Facultad de Ciencias Naturales y Exactas

Universidad del Valle

\title{
A simple test for asymptotic stability in some dynamical systems
}

\author{
Eduardo Ibargüen Mondragón Miller Cerón Gómez \\ Universidad del Nariño \\ Jhoana Patricia Romero Leiton \\ Universidad del Antioquia
}

Received: September 20, 2013

Accepted: November 23, 2013

Pag. 21-32

Abstract

In this paper we analyze asymptotic stability of the dynamical system $\dot{x}=f(x)$ defined by a $\mathcal{C}^{1}$ function $f: \mathcal{D} \rightarrow \mathbb{R}^{n}$ where $\mathcal{D} \in \mathbb{R}_{+}^{n}$ is an open set. We obtain a criterion of stability for the equilibrium solution $\bar{x} \in \mathcal{D}$ when the vector field $f$ satisfies a) $\partial_{i} f_{i}(\bar{x})<0$ and $\left.b\right)\left(\partial_{i} f_{i}(\bar{x})\right)^{-1} \partial_{i} f_{j}(\bar{x})+\left(\partial_{j} f_{j}(\bar{x})\right)^{-1} \partial_{j} f_{i}(\bar{x})>2$ for $i, j=1, \ldots, n$.

Keywords: ordinary differential equations, asymptotic stability, equilibrium solution.

\section{Introduction}

In 1892, A. M. Lyapunov developed his stability theory for nonlinear ordinary differential equations which characterizes the behavior of the dynamical systems trajectories in the sense that nearby solutions remain that way from now on (Hirsch and Smale, [9]). He established very useful stability criteria for dynamical systems of the form:

$$
\dot{x}=f(x),
$$

where $f: \mathcal{D} \rightarrow \mathbb{R}^{n}$ is a $\mathcal{C}^{1}$ map and $\mathcal{D} \subset \mathbb{R}^{n}$ is an open set.

The first Lyapunov method, also known as Indirect Method of Lyapunov (IML) allows to studying the stability of the equilibrium points for a dynamical system of the type (1) by analyzing the stability of the trivial solution for the linearized system:

$$
\frac{d y(t)}{d t}=D f(\bar{x}) y+G(y)
$$

where $G(y)=O\left(\|y\|^{2}\right)$. Using the IML, it is possible prove that $y=0$ is asymptotically stable if and only if $\Re(\lambda)<0$ for any eigenvalue $\lambda$ of the matrix $D f(\bar{x})$, and so unstable, if there exists an eigenvalue $\lambda$ of the matrix $D f(\bar{x})$ with $\mathfrak{R}(\lambda)>0$. We note that the IML does not allow us to obtain a conclusion if one of the eigenvalues $\lambda$ of the matrix $D f(\bar{x})$ has real part zero, $\mathfrak{R}(\lambda)=0$ (Khalil, [12]). 
In this paper, we will consider the second Lyapunov method, also known as Direct Method of Lyapunov (DML), in which the stability of an equilibrium point $\bar{x}$ requires the flow associated with the dynamical system (1) being decreased on some scalar function $V$ for which $\bar{x}$ is an isolated minimum. This function is known as the Lyapunov function.

For the Lyapunov function $V: \mathcal{D} \subseteq \mathbb{R}^{n} \rightarrow \mathbb{R}$ where $\mathcal{D}$ containing the origin, and its orbital derivative $\dot{V}: \mathcal{D} \subseteq \mathbb{R}^{n} \rightarrow \mathbb{R}$ defined by

$$
\dot{V}(x)=D V(x)(f(x)),
$$

the DML establishes:

1. If $V(x)$ is positive definite and $\dot{V}(x)$ is negative semi-definite, then the origin is stable.

2. If $V(x)$ is positive definite and $\dot{V}(x)$ is negative definite, then the origin is asymptotically stable.

In general, for any equilibrium solution of (1) the DML states that:

Theorem 1. Let $\bar{x} \in \mathcal{D}$ be an equilibrium of (1). Let $V: \mathcal{B} \rightarrow \mathbb{R}$ be a continuous function defined on a neigborhood $\mathcal{B} \subset \mathcal{D}$ of $\bar{x}$, differentiable on $\mathcal{B}-\bar{x}$, such that

a) $V(\bar{x})=0$ and $V(x)>0$ if $x \neq \bar{x}$;

b) $\dot{V}(x) \leq 0$ in $\mathcal{B}-\bar{x}$,

then $\bar{x}$ is stable. Furthemore, if

c) $\dot{V}(x)<0$ in $\mathcal{B}-\bar{x}$,

then $\bar{x}$ is asymptotically stable.

In the twentieth century, the DML became in the principal tool to analyze global stability of dynamical systems applied to basic sciences and engineering. The main setback of this method is precisely to find a Lyapunov function, because there is not a systematic method for finding. The suggestion is to propose a function and check if this candidate satisfies the stability conditions (Perko, [20]).

While the intention of A. M. Lyapunov was to study movement stability (Taylor and Francis, [14]), the DML found a wide range of applications. For example, in problems related with automatic regulation and control of dynamical processes (Rouche et al., [21]; Vasilév, [25]; Yoshizawa, [26]; Artstein, [2]; Barbastin, [3]); in competition models (Goh, [7, 8]; Takeuchi, [23]); in SIR models (Mena-Lorca and Hethcote, [16]; Safi and Garba, [22]), in SIRS models (O'Regan et al., [19]); in models with two compartments (A. Yu, [1]), and in the proof of the Hopf bifurcation theorem (cited by O?Regan et al., [19]).

Recently, Lyapunov functions are being applied within the fractional calculus to analyze the stability of dynamical systems. In this field, the method is called 
Fractional Lyapunov Direct Method (Yan Li et al., [13]; Momani and Hadid, [17]; Zhang et al., [27]; Tarasov, [24]). In 2011, it was used Lyapunov functions to analyze the dynamics of the Hopf bifurcation in a class of models that exhibit Zip bifurcation (Escobar and Gonzáles, [4], Giesl and Hafstein, [5, 6]). In 2012 the same authors designed an algorithm to explain the construction of these functions.

There are some systems where the Lyapunov function is defined in a natural way, like in the case of electrical or mechanical systems where energy is often a Lyapunov function. In mathematical biology, more precisely in population dynamic modeled through the mass action law, the functions of Goh type

$$
V(x)=\sum_{i=1}^{n} a_{i}\left[x_{i}-\bar{x}_{i}-\bar{x}_{i} \ln \left(\frac{x_{i}}{\bar{x}_{i}}\right)\right],
$$

where $a_{i}$ for $i=1, \ldots, n$ are positive constants that satisfy the first item of Theorem 1 while the other items are reduced to find the constants $a_{i}$ that will satisfy them.

B. S. Goh (Goh, [7]) used the function defined in (2) to prove global stability in mutualism models of the form

$$
\dot{x}_{i}=x_{i} f_{i}\left(x_{1}, x_{2}, \ldots, x_{n}\right) \quad i=1,2, \ldots, n .
$$

In this paper we establish global stability properties for the dynamical system (1) following the same ideas of S. B. Goh in (Goh, [7]). That is, we use the Lyapunov function (2) with specific values of the constants $a_{i}$ to determine the stability conditions.

\section{Calculus and linear algebra}

Theorem 2. Let $E$ be an open subset of $\mathbb{R}^{n}$ containing $x_{0}$, if $f: E \subset \mathbb{R}^{n} \rightarrow \mathbb{R}$ such that $f \in \mathcal{C}^{3}(E), f\left(x_{0}\right)=0$ and Hessian matrix $H f\left(x_{0}\right)$ is positive definite, then $x_{0}$ is a relative minimum of $f$. Similarly, if $H f\left(x_{0}\right)$ is negative definite, then $x_{0}$ is a relative maximum of $f$.

See [15] for proof of Theorem 2.

Theorem 3. (Sylvester's Criterion). A real symmetric matrix is positive definite positive.

See [10] for proof of Theorem 3.

\section{Test of stability}

In this section we establish a test for the asymptotic stability of the system (1) equilibrium when $\mathcal{D}$ is an open subset of

$$
\mathbb{R}_{+}^{n}=\left\{\left(x_{1}, \ldots, x_{n}\right) \in \mathbb{R}^{n}: x_{i} \geq 0 \text { for } i=1, \ldots, n\right\} .
$$

The following proposition relates the equilibrium stability with the sign of certain determinants. 
Proposition 1. Let $\mathcal{D}$ be an open subset of $\mathbb{R}_{+}^{n}$ containing $\bar{x}=\left(\bar{x}_{1}, \ldots, \bar{x}_{n}\right)$. Suppose that the function $f: \mathcal{D} \rightarrow \mathbb{R}^{n}$ defined in (1) satisfies $f \in \mathcal{C}^{1}(\mathcal{D})$ and $f(\bar{x})=0$. Let $\Delta_{j}(\bar{x})$ be the determinants defined by

$$
\Delta_{j}(\bar{x})=(-1)^{j}\left|\frac{a_{j}}{\bar{x}_{j}} \frac{\partial f_{j}(\bar{x})}{\partial x_{i}}+\frac{a_{i}}{\bar{x}_{i}} \frac{\partial f_{i}(\bar{x})}{\partial x_{j}}\right|_{i=1, \ldots, j}, \quad j=1, \ldots, n
$$

where $a_{j}$ is a positive constant.

1. If $\Delta_{j}(\bar{x})$ for $j=1, \ldots, n$ are positive, then $\bar{x}$ is globally asymptotically stable.

2. If $\Delta_{j}(\bar{x})$ for $j=1, \ldots, n$ has alternate signs starting with a negative value, then $\bar{x}$ is unstable.

Proof. Let $a_{1}, \ldots, a_{n}$ be positive constants, for $\bar{x}=\left(\bar{x}_{1}, \ldots, \bar{x}_{n}\right) \in \mathcal{D}$, then the function defined in (2) satisfies the condition $V(\bar{x})=0$. On the other hand, the i-th term of (2) is:

$$
\eta\left(x_{i}\right)=a_{i}\left[x_{i}-\bar{x}_{i}-\bar{x}_{i} \ln \left(\frac{x_{i}}{\bar{x}_{i}}\right)\right]
$$

Observe that

$$
\eta^{\prime}\left(x_{i}\right)=a_{i}\left(1-\frac{\bar{x}_{i}}{x_{i}}\right)
$$

Observe that which implies that $\eta^{\prime}\left(x_{i}\right)>0$ if and only if $\bar{x}_{i}<x_{i}$ and $\eta^{\prime}\left(x_{i}\right)<0$ if and only if $\bar{x}_{i}>x_{i}$. Thus $\bar{x}_{i}$ is a global minimum of $\eta$ defined in (4). Since $\eta\left(\bar{x}_{i}\right)=0$, then $\eta\left(x_{i}\right)>0$ for all $x_{i} \neq \bar{x}_{i}$ therefore $V(x)>0$ for all $x \neq \bar{x}$. From DML we conclude that if its orbital derivative is negative $(\dot{V}(x)<0)$ for all $x \in \mathcal{D} \backslash\{\bar{x}\}$, then $\bar{x}$ is asymptotically stable on $\mathcal{D}$, while $\bar{x}$ is unstable when $\dot{V}(x)>0$ (see Theorem 1).

Observe that $\dot{V}(x)=-g(x)$ where

$$
g(x)=\sum_{i=1}^{n} a_{i}\left(\frac{\bar{x}_{i}}{x_{i}}-1\right) f_{i}(x) .
$$

Since $g(\bar{x})=0$, then to prove the stability of $\bar{x}$ it is enough to verify that $\bar{x}$ is a minimum of $g$ on $\mathcal{D}$, and any other equilibrium solution $y \in \mathcal{D}$ of (1) satisfies that $g(y) \geq g(\bar{x})$. The derivative of $g$ is given by the gradient vector

$$
\nabla g(x)=\left(\frac{\partial g(x)}{\partial x_{1}}, \ldots, \frac{\partial g(x)}{\partial x_{n}}\right)
$$

where

$$
\frac{\partial g(x)}{\partial x_{k}}=-a_{k} \frac{\bar{x}_{k}}{x_{k}^{2}} f_{k}(x)+\sum_{i=1}^{n} a_{i}\left(\frac{\bar{x}_{i}}{x_{i}}-1\right) \frac{\partial f_{i}(x)}{\partial x_{k}},
$$

for $k=1, \ldots, n$. From (5) we have that $\partial g(\bar{x}) / \partial x_{k}=0$ for $k=1, \ldots, n$, which implies $\nabla g(\bar{x})=0$. Therefore $\bar{x}$ is a critical point of $g$. 
The Hessian matrix of $g(x)$ is

$$
H g(x)=\left(\frac{\partial^{2} g(x)}{\partial x_{i} \partial x_{j}}\right)_{i, j=1, \ldots, n},
$$

where

$$
\begin{aligned}
\frac{\partial^{2} g(x)}{\partial x_{j} \partial x_{k}} & =-a_{k} \frac{\bar{x}_{k}}{x_{k}^{2}} \frac{\partial f_{k}(x)}{\partial x_{j}}+\frac{\partial}{\partial x_{j}}\left[\sum_{i=1}^{n} a_{i}\left(\frac{\bar{x}_{i}}{x_{i}}-1\right) \frac{\partial f_{i}(x)}{\partial x_{k}}\right] \\
& =-\left(a_{k} \frac{\bar{x}_{k}}{x_{k}^{2}} \frac{\partial f_{k}(x)}{\partial x_{j}}+a_{j} \frac{\bar{x}_{j}}{x_{j}^{2}} \frac{\partial f_{j}(x)}{\partial x_{k}}\right)+\sum_{i=1}^{n} a_{i}\left(\frac{\bar{x}_{i}}{x_{i}}-1\right) \frac{\partial^{2} f_{i}(x)}{\partial x_{j} \partial x_{k}},
\end{aligned}
$$

for $j, k=1, \ldots, n$ and $j \neq k$. As a result

$$
\frac{\partial^{2} g(\bar{x})}{\partial x_{j} \partial x_{k}}=-\left(\frac{a_{k}}{\bar{x}_{k}} \frac{\partial f_{k}(\bar{x})}{\partial x_{j}}+\frac{a_{j}}{\bar{x}_{j}} \frac{\partial f_{j}(\bar{x})}{\partial x_{k}}\right) .
$$

Therefore, the determinant of Hessian matrix of $g(x)$ evaluated at $\bar{x}$ is:

$$
\begin{aligned}
|H g(\bar{x})| & =\left|\frac{\partial^{2} g(x)}{\partial x_{i} \partial x_{j}}\right|_{i, j=1, \ldots, n} \\
& =\left|-\left(\frac{a_{j}}{\bar{x}_{j}} \frac{\partial f_{j}(\bar{x})}{\partial x_{i}}+\frac{a_{i}}{\bar{x}_{i}} \frac{\partial f_{i}(\bar{x})}{\partial x_{j}}\right)\right|_{i, j=1, \ldots, n} \\
& =(-1)^{j}\left|\frac{a_{j}}{\bar{x}_{j}} \frac{\partial f_{j}(\bar{x})}{\partial x_{i}}+\frac{a_{i}}{\bar{x}_{i}} \frac{\partial f_{i}(\bar{x})}{\partial x_{j}}\right|_{i, j=1, \ldots, n} \\
& =\Delta_{n}(\bar{x}) .
\end{aligned}
$$

Since $H g(\bar{x})$ is a symmetric matrix, and assuming that all its principal minors are positive, then from Theorem 3 we have that $\bar{x}$ is a local minimum of $g$ on $\mathcal{D}$. Now, suppose that $y \in \mathcal{D}$ is another equilibrium solution of $(1)$ then $g(y)=g(\bar{x})=0$. Similarly it is verified that if its principal minors have alternating signs for $k=1, \ldots, n$, starting with a negative value, then $\bar{x}$ is unstable, which completes the proof.

From the above proposition, the following corollary is derived:

Corollary 4. If the Hessian matrix $H g(x)$ defined in (6) evaluated at $\bar{x}$ is positive definite, then $\bar{x}$ is globally asymptotically stable on $\mathcal{D}$ and unstable when $\mathrm{Hg}(\bar{x})$ is negative definite.

The following theorem summarizes the main result of this work. The novelty of next test consists in replacing the expertise of the authors to find the constants $a_{i}$ defined in (2) for conditions easy to verify.

Theorem 5 (Stability Test). Let $\bar{x} \in \mathcal{D} \subset \mathbb{R}_{+}^{n}$ be an equilibrium solution of nonlinear system (1). If 
1. $\frac{\partial f_{i}(\bar{x})}{\partial x_{i}}<0$ for $i=1,2, \ldots, n$. and

2. $l_{i j}(\bar{x})>2$ for $i, j=1, \ldots, n$ with $i \neq j$, where

$$
l_{i j}(\bar{x})=\left(\frac{\partial f_{i}(\bar{x})}{\partial x_{i}}\right)^{-1} \frac{\partial f_{i}(\bar{x})}{\partial x_{j}}+\left(\frac{\partial f_{j}(\bar{x})}{\partial x_{j}}\right)^{-1} \frac{\partial f_{j}(\bar{x})}{\partial x_{i}},
$$

then $\bar{x}$ is globally asymptotically stable.

Proof. Let $x=\left(x_{1}, \ldots, x_{n}\right) \in \mathcal{D}$, we will prove that the quadratic form

$$
\begin{aligned}
G(\bar{x}, x) & =x^{T} H g(\bar{x}) x \\
& =\sum_{j=1}^{n}\left(\sum_{k=1}^{n} \frac{\partial^{2} g(\bar{x})}{\partial x_{j} \partial x_{k}} x_{j} x_{k}\right),
\end{aligned}
$$

is positive. Since

$$
\frac{\partial^{2} g(\bar{x})}{\partial x_{j} \partial x_{k}}=\frac{\partial^{2} g(\bar{x})}{\partial x_{k} \partial x_{j}}
$$

for $j, k=1, \ldots, n$, then (9) is rewritten as:

$$
\begin{aligned}
G(\bar{x}, x)= & \sum_{k=1}^{n} \frac{\partial^{2} g(\bar{x})}{\partial x_{k}^{2}} x_{k}^{2}+2 \sum_{k=2}^{n} \frac{\partial^{2} g(\bar{x})}{\partial x_{1} \partial x_{k}} x_{1} y_{k}+2 \sum_{k=3}^{n} \frac{\partial^{2} g(\bar{x})}{\partial x_{2} \partial x_{k}} x_{2} y_{k} \\
& +\cdots+2 \frac{\partial^{2} g(\bar{x})}{\partial x_{n-1} \partial x_{n}} x_{n-1} x_{n} .
\end{aligned}
$$

Substituting (8) in (10) we have:

$$
\begin{aligned}
G(\bar{x}, x)= & -\sum_{k=1}^{n} 2 \frac{a_{k}}{\bar{x}_{k}} \frac{\partial f_{k}(\bar{x})}{\partial x_{k}} x_{k}^{2}-\sum_{k=2}^{n} 2\left(\frac{a_{k}}{\bar{x}_{k}} \frac{\partial f_{k}(\bar{x})}{\partial x_{1}}+\frac{a_{1}}{\bar{x}_{1}} \frac{\partial f_{1}(\bar{x})}{\partial x_{k}}\right) y_{1} x_{k} \\
& -\sum_{k=3}^{n} 2\left(\frac{a_{k}}{\bar{x}_{k}} \frac{\partial f_{k}(\bar{x})}{\partial x_{2}}+\frac{a_{2}}{\bar{x}_{2}} \frac{\partial f_{2}(\bar{x})}{\partial x_{k}}\right) x_{2} x_{k}+\cdots+ \\
& -2\left(\frac{a_{n}}{\bar{x}_{n}} \frac{\partial f_{n}(\bar{x})}{\partial x_{n-1}}+\frac{a_{n-1}}{\bar{x}_{n-1}} \frac{\partial f_{n-1}(\bar{x})}{\partial x_{n}}\right) x_{n-1} x_{n}
\end{aligned}
$$

Let

$$
a_{k}=-\bar{x}_{k}\left[2 \frac{\partial f_{k}(\bar{x})}{\partial x_{k}}\right]^{-1}, k=1, \ldots, n
$$


Substituting (12) in (11) we have:

$$
\begin{aligned}
G(\bar{x}, x)= & \sum_{k=1}^{n} x_{k}^{2}+\sum_{k=2}^{n}\left[\left(\frac{\partial f_{k}(\bar{x})}{\partial x_{k}}\right)^{-1} \frac{\partial f_{k}(\bar{x})}{\partial x_{1}}+\left(\frac{\partial f_{1}(\bar{x})}{\partial x_{1}}\right)^{-1} \frac{\partial f_{1}(\bar{x})}{\partial x_{k}}\right] x_{1} x_{k} \\
& +\sum_{k=3}^{n}\left[\left(\frac{\partial f_{k}(\bar{x})}{\partial x_{k}}\right)^{-1} \frac{\partial f_{k}(\bar{x})}{\partial x_{2}}+\left(\frac{\partial f_{2}(\bar{x})}{\partial x_{2}}\right)^{-1} \frac{\partial f_{2}(\bar{x})}{\partial x_{k}}\right] x_{2} x_{k} \\
& +\cdots+\left[\left(\frac{\partial f_{n}(\bar{x})}{\partial x_{n}}\right)^{-1} \frac{\partial f_{n}(\bar{x})}{\partial x_{n-1}}+\left(\frac{\partial f_{n-1}(\bar{x})}{\partial x_{n-1}}\right)^{-1} \frac{\partial f_{n-1}(\bar{x})}{\partial x_{n}}\right] x_{n-1} x_{n} \\
> & \sum_{k=1}^{n} x_{k}^{2}+\sum_{k=2}^{n} 2 x_{1} x_{k}+\sum_{k=3}^{n} 2 x_{2} x_{k}+\cdots+2 x_{n-1} x_{n} \\
= & \left(x_{1}+x_{2}+\cdots+x_{n}\right)^{2} \\
> & 0 .
\end{aligned}
$$

In consequence, from Corollary 4 we conclude that $\bar{x}$ is globally asymntoticallv stable in $\mathcal{D}$.

\section{Application of main result}

In this section we will apply the Theorem 5 to prove the asymptotic stability of nontrivial equilibrium of the nonlinear system

$$
\frac{d x_{j}}{d t}=\alpha_{j} x_{j}\left(1-x_{j}\right)-\sigma_{j} \prod_{i=1}^{n} x_{i}, \quad j=1,2, \ldots, n,
$$

where $0<\alpha_{j}<1$ and $0<\sigma_{j}<1$ for $j=1,2, \ldots, n$. Our set of interest is:

$$
\mathcal{D}_{1}=\left\{x \in \mathbb{R}^{n}: 0 \leq x_{i} \leq 1, i, j=1,2, \ldots, n\right\} .
$$

The following lemma ensures that all solutions of (14) starting in $\mathcal{D}_{1}$ remain there for all $t \geq 0$.

Lemma 6. The set $\mathcal{D}_{1}$ defined in (15) is positively invariant for the solutions of the system (14).

Proof. Let $x=\left(x_{1}^{0}, x_{2}^{0}, \ldots, x_{n}^{0}\right)$ be given. If there is $1 \leq j \leq n$ such that $x_{j}^{0}=0$, then we see directly from the unique and existent result that $x_{j}(t) \equiv 0$ for all $t \geq 0$, and so for $k \neq j$ such that $x_{k}^{0} \neq 0$, we have that $x_{k}(t)$ satisfies the logic differential equation:

$$
\frac{d x_{k}}{d t}=\alpha_{k} x_{k}\left(1-x_{k}\right)
$$

for which we know that $0 \leq x_{k}(t) \leq 1$. In other words, if there is $1 \leq j \leq n$ such that $x_{j}^{0}=0$, we have that $0 \leq x_{k}(t) \leq 1$ for $1 \leq k \leq n$. Now, we assume that $x=\left(x_{1}^{0}, x_{2}^{0}, \ldots, x_{n}^{0}\right) \in \mathcal{D}_{1}$ is such that $x_{j}^{0} \neq 0$ for any $1 \leq j \leq n$. In this case, we know that $x_{j}(t)$ for any $t \geq 0$ and $0 \leq j \leq n$. Then, from (14) we obtain:

$$
\frac{d x_{j}}{d t} \leq \alpha_{j} x_{j}\left(1-x_{j}\right), \quad j=1,2, \ldots, n
$$


or equivalently

$$
-x_{j}^{-2} \frac{d x_{j}}{d t}+\alpha_{j} x_{j}^{-1} \geq \alpha_{j}, \quad j=1,2, \ldots, n .
$$

Let $z=x_{j}^{-1}$, then $d z / d t=-x_{j}^{-2} d x_{j} / d t$. Substituting $z$ and $d z / d t$ in (16) we have

$$
\frac{d z}{d t}+\alpha_{j} z \geq \alpha_{j}
$$

Multiplying the above inequality by $e^{\alpha_{j} t}$ we obtain:

$$
\frac{d\left(e^{\alpha_{j} t} z\right)}{d t} \geq \alpha_{j} e^{\alpha_{j} t}
$$

Integrating the inequality (17) between 0 and $t$ we have:

$$
z(t) \geq 1+(z(0)-1) e^{-\alpha_{j} t} .
$$

Substituting $z=x_{j}^{-1}$ in (18) we obtain:

$$
x_{j}(t) \leq \frac{1}{1+\left[x_{j}^{-1}(0)-1\right] e^{-\alpha_{j} t}} .
$$

Therefore, we conclude that:

$$
0 \leq x_{j}(t) \leq 1 \text { for all } t \geq 0
$$

meaning $x=\left(x_{1}^{0}, x_{2}^{0}, \ldots, x_{n}^{0}\right) \in \mathcal{D}_{1}$ as desired.

The next proposition summarizes existent results of the equilibrium solutions of (14).

Proposition 2. The system (14) has at least $2^{n+1}-1$ equilibrium solution in $\mathcal{D}_{1}$.

Proof. The equilibrium solutions of (14) are given by the solutions of the algebraic system

$$
\alpha_{j} x_{j}\left(1-x_{j}\right)-\sigma_{j} \prod_{i=1}^{n} x_{i}=0, \quad j=1,2, \ldots, n .
$$

Observe that in the following cases, a) $\left.x_{j}=0, \mathrm{~b}\right) x_{j}=1$ and $x_{k}=0$ for $j \neq k$, the equations (19) are satisfied, which implies the existence of $2^{n}-1$ equilibrium of the form $x_{0}=\left(p_{1}, \ldots, p_{n}\right)$ where $p_{j}=0$ or $p_{j}=1$. On the other hand, from (19) we obtain:

$$
\frac{\alpha_{j}}{\sigma_{j}} x_{j}\left(1-x_{j}\right)=k, \quad j=1,2, \ldots, n,
$$

where $k=\prod_{i=1}^{n} x_{i}$. The solutions of (20) are:

$$
x_{j}=\frac{1 \pm \sqrt{1-4 k \sigma_{j} / \alpha_{j}}}{2}, \quad j=1,2, \ldots, n .
$$

The above implies that $x_{j}>0$ if and only if $0<k<\alpha_{n} / 4 \sigma_{n}$. Therefore, there are at least two equilibriums in $\operatorname{int}\left(\mathcal{D}_{1}\right)$. This completes the proof. 
The following proposition summarizes stability results of the equilibrium of (14).

Proposition 3. Suppose that the system (14) has an interior steady state $\bar{x} \in$ $\mathcal{D}_{2} \subset \mathcal{D}_{1}$ where

$$
\mathcal{D}_{2}=\left\{x \in \mathbb{R}^{n}: 0 \leq x_{i} \leq 1,0 \leq x_{i}+x_{j}, \leq 1 i, j=1,2, \ldots, n\right\} .
$$

then this steady state is globally asymptotically stable on the interior set of $\mathcal{D}_{1}$.

Proof. From (14) we conclude that:

$$
f_{j}(x)=\alpha_{j} x_{j}\left(1-x_{j}\right)-\sigma_{j} \prod_{k=1}^{n} x_{k}, \quad j=1,2, \ldots, n,
$$

which implies that

$$
\frac{\partial f_{j}(\bar{x})}{\partial \bar{x}_{j}}=\alpha_{j}\left(1-\bar{x}_{j}\right)-\alpha_{j} \bar{x}_{j}-\sigma_{j} \prod_{k=1, k \neq j}^{n} \bar{x}_{k}, \quad j=1,2, \ldots, n .
$$

From equilibrium equations we have:

$$
\alpha_{j}\left(1-\bar{x}_{j}\right)-\sigma_{j} \prod_{k=1, k \neq j}^{n} \bar{x}_{k}=0, \quad j=1,2, \ldots, n .
$$

Therefore, substituting (22) in (21) we verify the first hypothesis of Theorem 5, that is

$$
\frac{\partial f_{j}(\bar{x})}{\partial \bar{x}_{j}}=-\alpha_{j} \bar{x}_{j}<0, \quad j=1,2, \ldots, n .
$$

On the other hand,

$$
\begin{aligned}
l_{i j}(\bar{x}) & =\left(\frac{\partial f_{i}(\bar{x})}{\partial x_{i}}\right)^{-1} \frac{\partial f_{i}(\bar{x})}{\partial x_{j}}+\left(\frac{\partial f_{j}(\bar{x})}{\partial x_{j}}\right)^{-1} \frac{\partial f_{j}(\bar{x})}{\partial x_{i}} \\
& =\left(-\alpha_{i} \bar{x}_{i}\right)^{-1}\left(-\sigma_{i} \prod_{k=1, k \neq j}^{n} \bar{x}_{k}\right)+\left(-\alpha_{j} \bar{x}_{j}\right)^{-1}\left(-\sigma_{j} \prod_{k=1, k \neq i}^{n} \bar{x}_{k}\right) \\
& =\left(\frac{\sigma_{i}}{\alpha_{i}}+\frac{\sigma_{j}}{\alpha_{j}}\right) \prod_{k=1, k \neq i, k \neq j}^{n} \bar{x}_{k} .
\end{aligned}
$$

From (22) we have:

$$
\frac{\sigma_{j}}{\alpha_{j}}=\frac{1-\bar{x}_{j}}{\prod_{k=1, k \neq j}^{n} \bar{x}_{k}}, \quad j=1,2, \ldots, n .
$$

Substituting (24) in (23) we obtain:

$$
l_{i j}(\bar{x})=\frac{1-\bar{x}_{i}}{\bar{x}_{j}}+\frac{1-\bar{x}_{j}}{\bar{x}_{i}}, \quad \text { for } i \neq j .
$$

From hypothesis $\bar{x} \in \mathcal{D}_{2}$, results that $0<\bar{x}_{i}+\bar{x}_{j}<1$ which implies $\left(\bar{x}_{i}+\bar{x}_{j}\right)^{2}<$ $\bar{x}_{i}+\bar{x}_{j}$, or equivalently $\left(1-\bar{x}_{i}\right) \bar{x}_{i}+\left(1-\bar{x}_{j}\right) \bar{x}_{j}>2 \bar{x}_{i} \bar{x}_{j}$. The above implies that the second hypothesis of Theorem 5 is satisfied. That is $l_{i j}>2$. Therefore $\bar{x}$ is globally asymptotically stable on interior set of $\mathcal{D}_{1}$. 


\subsection{Numerical solutions}

One of the possible applications for system (14) when $n=3$ could be the competition among three species with logistic growth. The simulation of Figure 1 was made with the following data: $\alpha_{1}=0.1, \alpha_{2}=0.2, \alpha_{3}=0.15, \sigma_{1}=0.08$, $\sigma_{2}=0.15$ and $\sigma_{3}=0.14$. In this case the solutions of (14) tend to the coexistent equilibrium $P_{1}=(0.68,0.72,0.53)$ which agrees with the theoretical results.

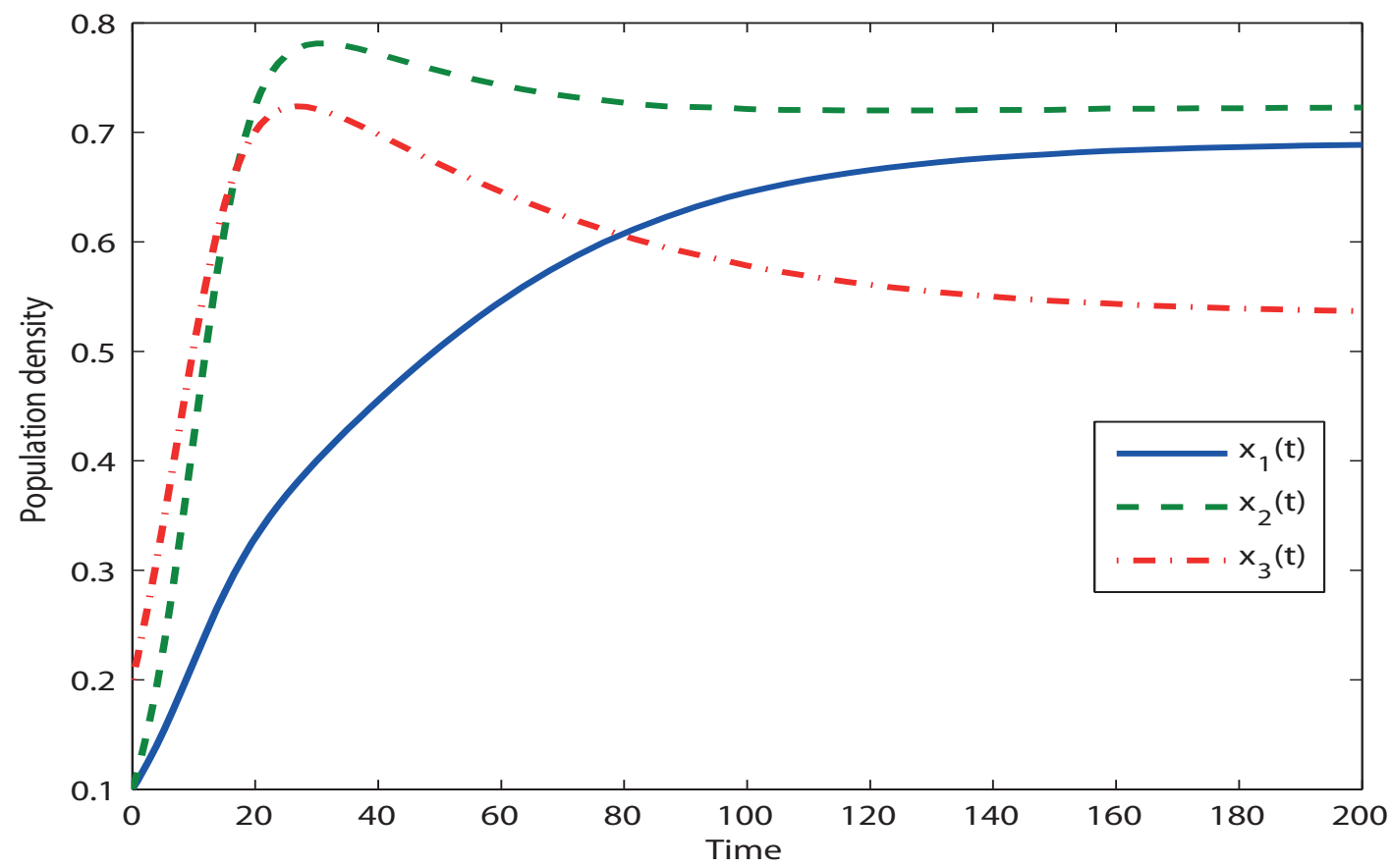

Figure 1: Graphs of the component solutions $x_{1}, x_{2}$ and $x_{3}$ of (14) for $n=3$. In this case, $\alpha_{1}=0.1, \alpha_{2}=0.2, \alpha_{3}=0.15, \sigma_{1}=0.08, \sigma_{2}=0.15, \sigma_{3}=0.14$.

\section{Conclusión}

In certain areas of applied mathematics such as Biomathematics, the qualitative analysis of the solutions of dynamical systems defined by ordinary differential equations is fundamental to understand problems in biology (Ibargüen et al. [11]). In this sense, the DML is very practical and widely used to analyze the stability of dynamical systems. In this article we use the DML to establish easier conditions to verify the assurance of global asymptotic stability of the equilibrium solutions of some dynamical systems. The fact that these conditions are defined in terms of $\partial f_{i}(\bar{x}) / \partial x_{i}$ for $i=1, \ldots, n$, suggest the possibility that the stability test (Theorem 5) can be used to numerically verify asymptotic stability.

\section{Acknowledgements}

We want to thank to anonymous referees and Dr. L. Esteva for their valuable comments and suggestions that helped us to improve the paper. E. Ibarguen acknowledges support from project No 082-16/08/2013 (VIPRI-UDENAR). 


\section{References}

[1] Alexandrov A. Y. (2003). On the Construction of Lyapunov Functions for Nolinear System, Differential Equations, 41 (3), 303-309.

[2] Artstein Z. (1978). Uniform Asymptotic Stability via the Limiting Equations, J. Diff. Equat., 27 (2), 172-189.

[3] Barbashin E. A. (1970). Lyapunov Functions, Nauka, Moscow. [in Russian].

[4] Escobar C. and Gonzáles J. (2011). Dinámica de la bifurcación de Hopf en una clase de modelos de competencia que exhiben la bifurcación Zip, Revista de Ingeniería Universidad de Medellín, 10 (19), 159-169.

[5] Giesl P. and Hafstein S. (2010). Existence of Piecewise Affine Lyapunov Functions in two Dimensions, J. Math. Anal. Appl., 371, 233-248.

[6] Giesl P. and Hafstein S. (2012). Construction of Lyapunov Functions for Nonlinear Planar Systems by Linear Programming, Journal of Mathematical Analysis and Applications, 388, 463-479 .

[7] Goh B. S. (1979). Stability in Models of Mutualism, The American Naturalist, The American Society of Naturalists, 113 (2) 261-275.

[8] Goh B. S. (1980). Management and Analysis of Biological Populations, Elsevier Science. Amsterdam.

[9] Hirsch M. and Smale S. (1974). Differential Equations-Dynamical Systems and Linear Algebra, Academic Press, New York.

[10] Hoffman K. and Kunze R. (1971). Linear Algebra, 2nd ed., Prentice-Hall, Englewood Cliffs, New Jersey.

[11] Ibargüen-Mondragón E., Esteva L. and Chávez-Galán L., (2011). A Mathematical Model for Cellular Immunology of tuberculosis, J. Mathematical Biosciences and Engineering, 8 (4), 973-986.

[12] Khalil H. (1996). Nolinear System, 2nd editions, Prentice-Hall, London.

[13] Li Y., Chen Y. and Podlubny I. (2010). Stability of Fractional-order Nonlinear Dynamic Systems: Lyapunov Direct Method and Generalized Mittag-Leffler Stability, Computers and Mathematics with Applications, 59, 1810-1821.

[14] Lyapunov A. M. (1992). The General Problem of the Stability of Motion, Taylor and Francis. London.

[15] Marsden J. and Tromba A. (1976). Vector Calculus, W. H. Freeman and Company, New York.

[16] Mena-Lorca J. and Hethcote H. W. (1992). Dynamics Model of Infectious Diseases as Regulator of Populations Sizes, J. Math Biol, 30 693-716, 
[17] Momani S. and Hadid S. (2004). Lyapunov Stability Solutions of Fractional Integer-differential Equations. International Journals of Mathematics and Mathematical Sciences, 47, 2503-2507.

[18] Ogata K. (1990). Moderm Control Engineering, 2nd edition, Prentice-Hall, London.

[19] O'Regan S. M., Kelly T. C., Korobenikov A., O'Callaghan M. J. A. and Pokrovskii A. V. (2009). Lyapunov Functions for SIR and SIRS Epidemic Models, Applied mathematics Letter.

[20] Perko L. (1991).Differential Equations and Dynamical Systems, Springer, New York.

[21] Rouche N., Habets P. and Laloy M. Stability Theory by Liapunov's Direct Method, Springer, N. Y., (1977); Mir, Moscow, (1980).

[22] Safi M. A. and Garba S. (2012).Global Stability Analysis of SEIR Model with Holling Type II Incidence Function; Computational and Mathematical Methods in Medicine; Hindawi Publishing Corporation.

[23] Takeuchy Y. (1996). Global Dynamical Properties of Lotka-Volterra System, World Scientific, Singapore.

[24] Tarasov V. E. (2007). Fractional Stability; Available online: http://arxiv.org/abs/0711.2117v1.

[25] Vasilév S. N. (1981). The Comparison Method in the Mathematical Theory of Systems. Uravneniya 17 (9), 1562-1573.

[26] Yoshizawa T. (1966). Stability Theory by Liapunov's Second Method, Publ. Math. Soc. Japan, Tokyo.

[27] Zhang L., Li J. and Chen G. (2005). Extension of Lyapunov Second Method by Fractional calculus. Pure and Applied mathematics, 3, 1008-5313.

\section{Author's address}

Eduardo Ibargüen-Mondragón

Departamento de Matemática y Estadística, Universidad de Nariño,

San Juan de Pasto - Colombia

edbargun@udenar.edu.co

Miller Cerón Gómez

Departamento de Matemática y Estadística, Universidad de Nariño,

San Juan de Pasto - Colombia

millercg@udenar.edu.co

Jhoana Patricia Romero Leitón

Instituto de Matemáticas, Universidad de Antioquia, Medellín - Colombia

patirom3@udea.edu.co 